\title{
BMJ Open Application of the PRECEDE- PROCEED model in the development of evidence-informed interventions for drowning prevention: a mixed-methods study protocol
}

Jonathan P Guevarra (D) ," Amy E Peden (D) , ${ }^{2,3,4}$ Richard Charles Franklin (D) ${ }^{3,4}$

To cite: Guevarra JP, Peden AE, Franklin RC. Application of the PRECEDE-PROCEED model in the development of evidence-informed interventions for drowning prevention: a mixed-methods study protocol. BMJ Open 2021;11:e050688. doi:10.1136/ bmjopen-2021-050688

- Prepublication history and additional supplemental material for this paper are available online. To view these files, please visit the journal online. To view these files, please visit the journal online (http://dx.doi org/10.1136/bmjopen-2021 050688).

Received 26 February 2021 Accepted 02 July 2021

Check for updates

(C) Author(s) (or their employer(s)) 2021. Re-use permitted under CC BY-NC. No commercial re-use. See rights and permissions. Published by BMJ.

For numbered affiliations see end of article.

Correspondence to Jonathan P Guevarra; jpguevarra2@up.edu.ph

\section{ABSTRACT}

Introduction Drowning is a global public health threat, disproportionately impacting low-income and middleincome countries. In the Philippines, it is estimated that more than 5200 people die from drowning per annum. This number is likely to be higher than currently estimated with the inclusion of disaster-related and transportationrelated drowning. Drowning is preventable if appropriate preventive interventions are put in place which redress known risk factors.

Methods and analysis This study uses the PRECEDEPROCEED model (PPM), an eight-step health promotion planning and evaluation model for building and improving intervention programmes. This mixed-methods study, which can be used in any location, will be implemented in Los Baňos, Laguna, Philippines, identified as an area of concern for drowning. Using the PPM, data on drowning will be collected from death records, community observation, key informant interviews, focus group discussions and community survey. A range of analytical methods will be used to explore drowning data including univariate and $\chi^{2}$ analyses, analysis of variance, relative risk and calculating rates using population data. The quantitative data and themes drawn from qualitative data will be used to populate the first four phases of the PPM. Following the data collection, the remaining stages of the PPM will be designed and implemented in the barangay (village) with the highest drowning rate.

Ethics and dissemination This study has obtained ethical clearance from the University of the Philippines Manila Research Ethics Board (UPMREB 2017-425-01). Study findings will be disseminated through workshops and presentations to the local community as well as through peer-reviewed literature and conference presentations. The PPM has rarely been applied to drowning prevention and it is the aim that the study described in this protocol is expanded across other areas of the Philippines and to other countries with a high drowning burden to inform prevention efforts.

\section{INTRODUCTION}

The WHO has identified the need for an improved evidence base on drowning globally, particularly among low-income and
Strengths and limitations of this study

- This study uses a health promotion planning and evaluation model-the PRECEDE-PROCEED model and applies it to the issue of drowning.

- This study is being conducted in the Philippines, a low-income and middle-income country with significant loss of life and injury due to drowning.

- Although tested in a single site, the protocol describes a method which can be implemented across low-income, middle-income and high-income countries for the prevention of drowning.

- The model ensures the engagement with, and ownership of, the drowning prevention programme by the local community.

middle-income countries (LMICs). ${ }^{1}$ This need spans epidemiological data on the burden associated with drowning, risk factor identification and the development, implementation and evaluation of preventive strategies to better guide future effort and investment. ${ }^{2-5}$

Drowning is defined as the process of experiencing respiratory impairment from submersion or immersion in liquid with outcomes classified as death, morbidity or no morbidity. ${ }^{6}$ Across the full spectrum of drowning outcomes, ${ }^{7}$ a strengthened evidence base is required including improving data collection, quality and analyses.

Global estimates indicate 295000 people died from unintentional drowning in $2017 .{ }^{8}$ The true burden of fatal drowning is likely to be much higher as estimates exclude drowning deaths related to disasters and transportation. ${ }^{9}$ Even less is known about the non-fatal drowning burden globally, though work underway by the WHO aims to provide a standardised definition for the 
collection and assessment of non-fatal drowning and its outcomes. ${ }^{10}$

The global burden of injury and death from drowning is found in all economies and regions of the world, however LMICs account for over $90 \%$ of unintentional drowning deaths. ${ }^{11}$ Fatal drowning rates are highest in the African region, whereas over half of the world's drowning deaths occur in the Western Pacific and South-East Asian regions. ${ }^{8}$

Drowning is a significant issue in the Philippines, an archipelago of 7107 islands housing 108116615 people in the Western Pacific region. ${ }^{12}$ The Global Burden of Disease (GBD) study indicates 5245 people died from unintentional drowning in the Philippines in 2017, an increase of $5.4 \%$ on figures from $1990 .^{8}$ This represents an age-standardised cause-specific mortality rate of 5.3 per 100000 people. $^{8}$

Child drowning is also a significant issue in the Philippines. ${ }^{13}{ }^{14}$ In 2003, the Philippine National Injury Survey found for children after infancy (1-17 years), the rate of drowning mortality was $9.8 / 100000$; the next most common cause was road traffic injury $(9.1 / 100000) .{ }^{15}$ More recently, the GBD study estimated 2439 children $0-14$ years of age drowned in the Philippines in 2017. ${ }^{16}$ Such drowning estimates in the Philippines are likely to be significantly higher with the inclusion of disaster and transportation-related drownings, both key issues facing the Philippines as a nation. ${ }^{17-19}$

Detailed, country-level data on drowning are not currently available and data on non-fatal drowning are not routinely collected in the Philippines. Currently, the Online National Electronic Injury Surveillance System (ONEISS), a national injury (including drowning) surveillance system in the Philippines is in place. ONEISS data can be used as the source of information in determining primary cause and risk factors for drowning. ${ }^{16} 20$ However, there are limitations for using the ONEISS data as $^{1}$ the data are collected by selected hospitals, ${ }^{2}$ the system is web based and hospitals with no or poor access to the internet will have problems in using the system, ${ }^{3}$ drowning events captured by local health clinics are not usually reported, ${ }^{4}$ cataclysmic events and water transport incidents are not included, and, ${ }^{5}$ like other countries in Asia, misclassification of cases could be a problem. As such, a multisector action plan for drowning prevention in the Philippines identifies the need to strengthen drowning surveillance systems including disaggregated, regularly collected data and improving the evidence base through research. ${ }^{16}$

The WHO has identified 10 evidence-informed interventions and strategies designed to help drowningprevention practitioners-from non-governmental organisations and researchers to government officials and policy-makers-approach drowning prevention in a strategic, evidence-informed and multisectoral way. ${ }^{1}$ At a national or community level, drowning can be prevented through implementing six interventions and four crosscutting implementation strategies. ${ }^{1}$
There are a small number of studies reporting the effectiveness of drowning prevention interventions in LMICs, namely from Bangladesh ${ }^{21}$ and Thailand, ${ }^{22}$ however more work is required.

\section{The PRECEDE-PROCEED model (PPM)}

The PRECEDE-PROCEED model (PPM) was a ninephase health promotion planning and evaluation model first proposed by Green and Kreuter ${ }^{23}$ which provides a blueprint for building and improving intervention programmes. ${ }^{24}$ PRECEDE stands for Predisposing, Reinforcing and Enabling Constructs in Educational/Environmental Diagnosis and Evaluation. PROCEED stands for Policy, Regulatory and Organisational Constructs in Educational and Environmental Development. The PPM has since been updated to be an eight-phase model..$^{23}$ PRECEDE has four assessment and planning stages to guide the health promotion partners in selecting what problem to address, examining its underlying causes, and planning an intervention. PROCEED on the other hand includes four implementation and evaluation phases. ${ }^{25}$ The eight phases of the model are: phase 1-social assessment; phase 2—epidemiological assessment; phase 3-educational and ecological assessment; phase 4-administrative and policy assessment and intervention alignment; phase 5-implementation; phase 6-process evaluation; phase 7-impact evaluation and phase 8-outcome evaluation.

The PPM has been used previously in injury prevention in the realms of child pedestrian safety, ${ }^{26}$ child car restraint use, ${ }^{27}$ traffic safety behaviours among children ${ }^{28}$ and the prevention of musculoskeletal injury among nurses. ${ }^{29}$ The one study identified which used the PPM to evaluate a drowning prevention intervention, did so in a high-income setting. ${ }^{30}$ As such, the main objective of this study is to develop evidence-informed strategies on drowning prevention for LMICs using the PPM. Specifically, this study seeks to:

1. Determine the current situation of drowning in the selected site in terms of its contribution to morbidity and mortality.

2. Describe the behavioural, environmental and educational risk factors for drowning, and

3. Develop drowning prevention intervention and strategies based on the existing situation of drowning in the community.

Once intervention programme and strategies have been developed, implementation of these strategies will commence in the community site (phase 5 ). The evaluation phases are integral to any programme, and these will form part of the whole process. Process evaluation (phase 6), impact evaluation (phase 7) and outcome evaluation (phase 8) will follow the implementation of the programme.

\section{METHODS}

This is a mixed-methods study using convergent parallel design which aims to develop evidence-informed interventions for drowning prevention using the PPM. Both 

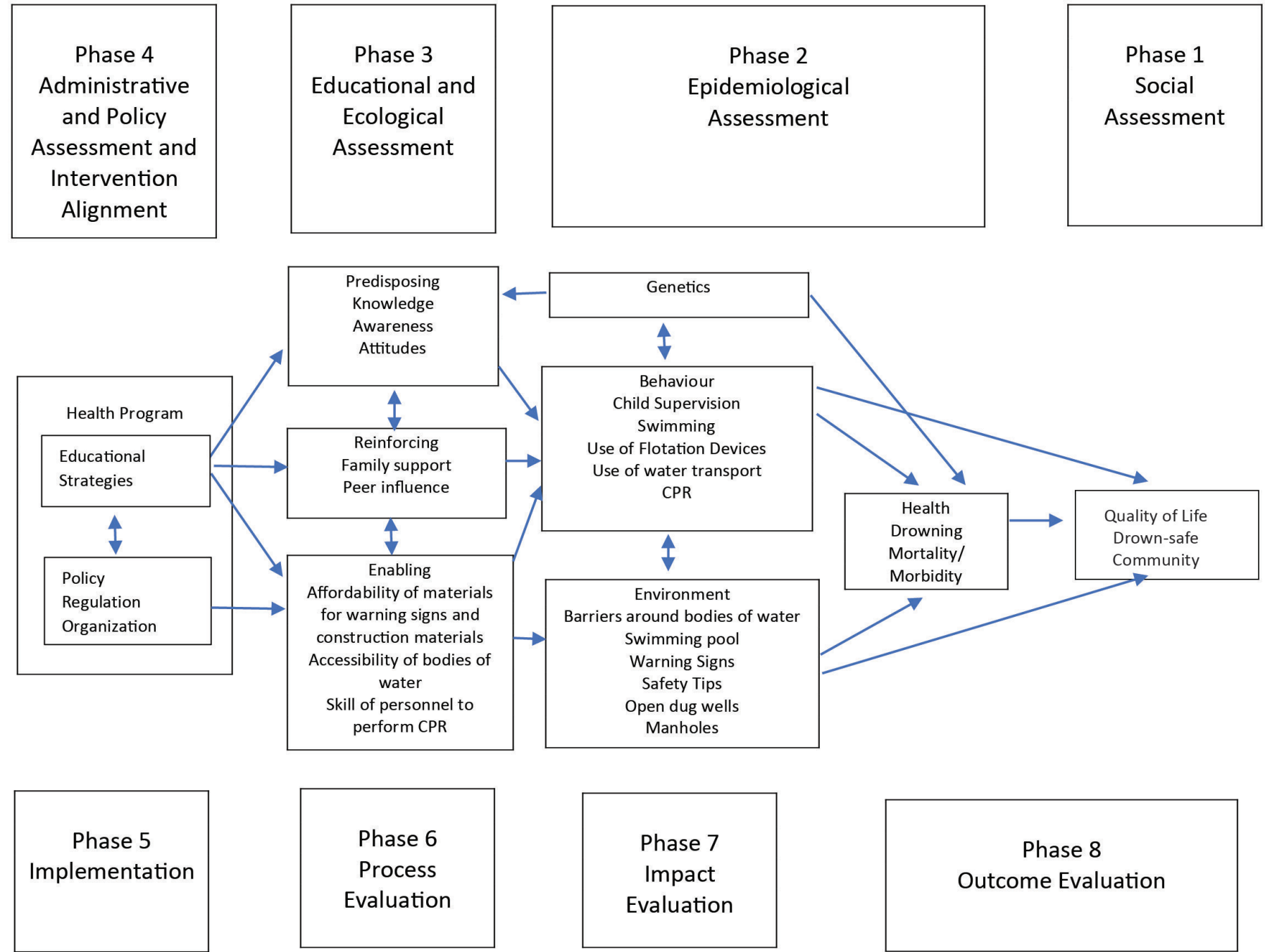

Figure 1 Application of the PRECEDE-PROCEED model (PPM) in this study. CPR, cardiopulmonary resuscitation.

quantitative and qualitative techniques will be used to gather the required data for the assessment phase of the PPM. The PRECEDE component of the PPM model, and how it will be used in this study, is depicted in figure 1. Table 1 shows the phases of the PPM, key questions and data sources for this study.

\section{Study site}

The process described in this protocol can be used for any location, however this research will be conducted in Los Baňos, Laguna (figure 2). Los Baňos, a town in the province of Laguna, is a popular tourist location in the Philippines containing many resorts. It is representative of a number of other similar sites in the Philippines and more broadly across Asia. However, we note that each location is unique and while the process described in the protocol can be applied across a range of locations, findings are likely to be location specific. A freshwater lake is also present at the study site which serves as one of the main sources of livelihood (eg, fishing) and as site for recreational activities. Several reports pointed to the area as a site of concern where drowning occurs which is worth investigating. ${ }^{31-33}$ Within Los Baňos, the barangay (village) with the highest drowning mortality rate will be identified as the site where quantitative and qualitative data collection will be done to determine the risk factors for drowning.

\section{Sampling design and population}

Engagement with the community is warranted in order to build trust and gather the most important data that will be needed in crafting drowning prevention interventions in the community. ${ }^{34}$ This involves courtesy calls with elected officials, community leaders and health sector officials and meetings with various groups engaged in water safety and drowning prevention efforts. Through engagement activities the purpose of the study, degree of involvement of various sectors and processes to be followed can be discussed and determined. In addition to this, a basic premise of the PPM is to include the target population and/or public health experts in every phase of assessment, planning, management, implementation and evaluation as this is a participatory health promotion model. $^{23}$

Purposive sampling will be undertaken for the key informant interviews (eight informants) and focus group 
Table 1 Steps 1-8 of the PPM model including key questions and sources of data

\begin{tabular}{|c|c|c|}
\hline Phase & Key questions to be addressed & Data sources \\
\hline 1. Social assessment & $\begin{array}{l}\text { What are the demographic characteristics of the } \\
\text { population whose quality of life you would like to } \\
\text { measure? } \\
\text { What are the social problems and perceived quality of } \\
\text { life of the target? }\end{array}$ & $\begin{array}{l}\text { Census data-Community Profile } \\
\text { Key informant interview } \\
\text { Focus group discussion }\end{array}$ \\
\hline 2. Epidemiological assessment & $\begin{array}{l}\text { What is impact in terms of death, disease, disability? } \\
\text { Is the problem susceptible to intervention? } \\
\text { Is the problem being/not being addressed by other } \\
\text { agencies in the community? } \\
\text { What is the potential yield if the problem is appropriately } \\
\text { addressed? } \\
\text { Is the problem highly ranked as a community priority? } \\
\text { What are the behavioural risk factors? } \\
\text { How important and changeable are these behavioural } \\
\text { risk factors? } \\
\text { What are the environmental causes of the health } \\
\text { concern? } \\
\text { How important and changeable are these environmental } \\
\text { causes of the health concern? }\end{array}$ & $\begin{array}{l}\text { Morbidity data } \\
\text { Mortality data } \\
\text { Key informant interview } \\
\text { Focus group discussion } \\
\text { Community survey } \\
\text { Observation }\end{array}$ \\
\hline
\end{tabular}

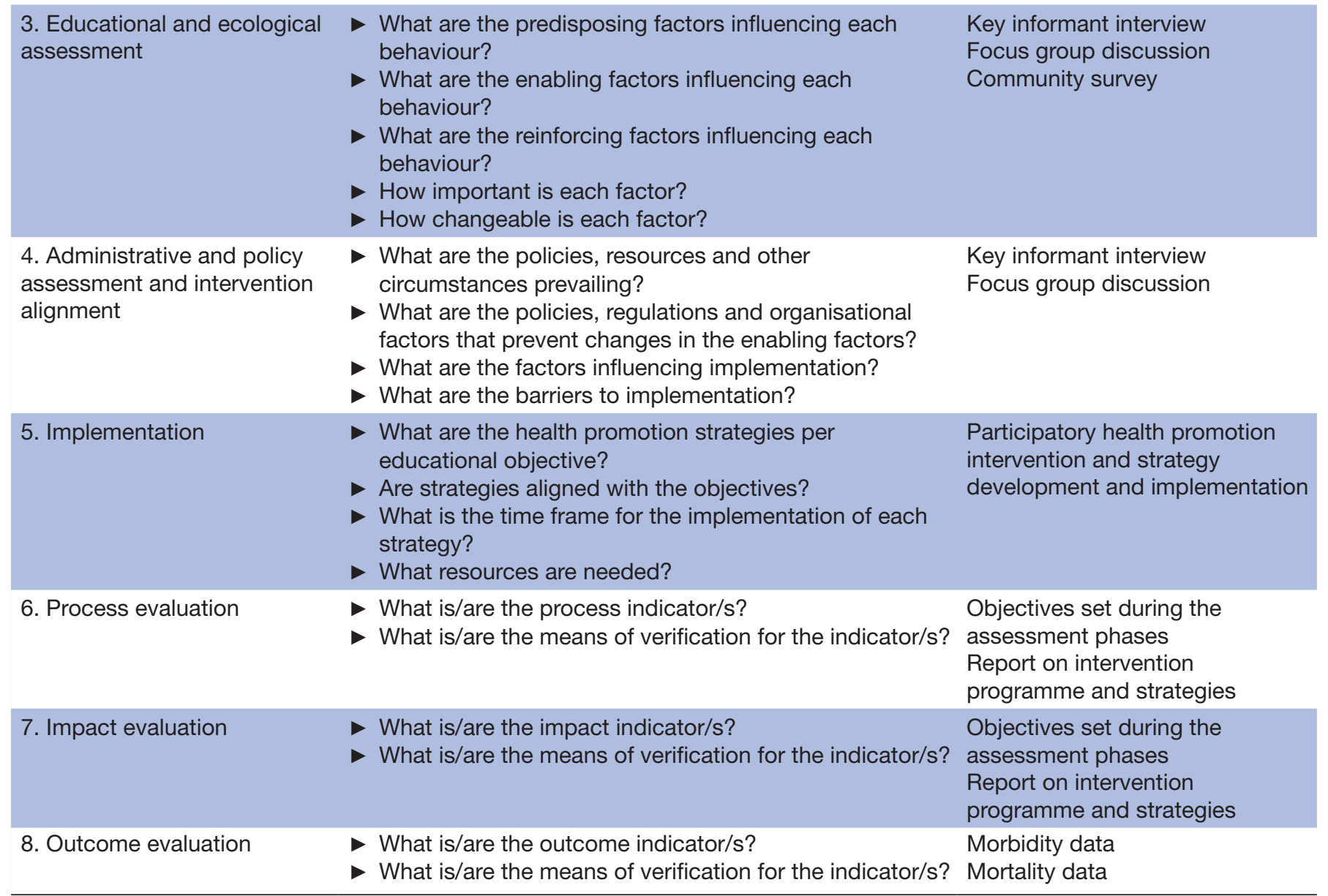

PPM, PRECEDE-PROCEED model.

discussions (four focus groups). Purposive sampling is a sampling technique in which the researcher relies on their own judgement when choosing members of the population to participate in the study. ${ }^{35}$ Purposive sampling is a non-probability sampling method and it occurs when elements selected for the sample are chosen by the judgement of the researcher. ${ }^{35}$ Key informants will be selected based on their knowledge of the drowning situation and involvement in drowning prevention activities in the study site. Key informants will include the city/ 


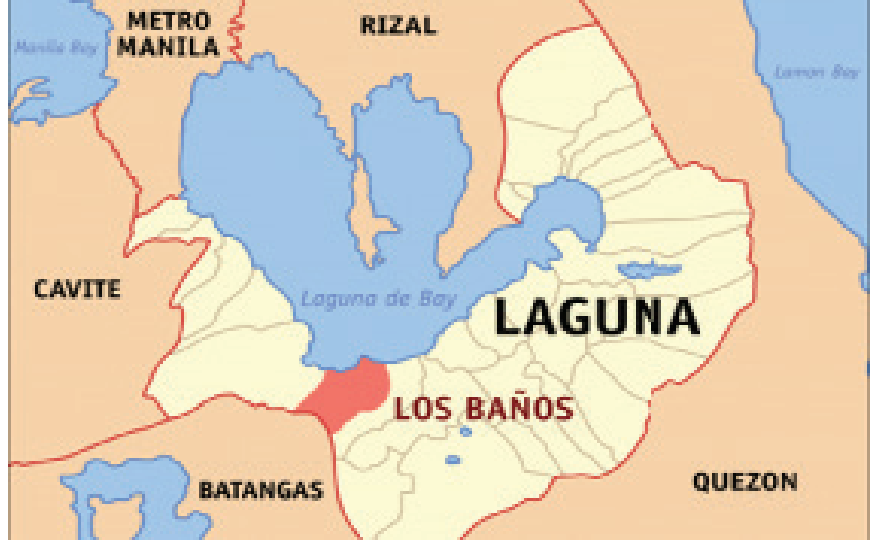

Figure 2 Map of Los Baňos, Laguna, Philippines. ${ }^{44}$

municipal health officer, drowning or injury prevention coordinator, informant from the hospital (handling injury cases), community leaders and the resort owners' association president. The key informant interview will be conducted in a room or area to be decided on by the informant with the consideration that the venue is free of environmental distraction or noise that can affect the conduct of the interview. Simultaneously, an environmental scan of policies and procedures relevant to drowning prevention will be undertaken and analysis of these documents will be conducted.

Participants in the focus group discussions will be selected from among the community health volunteers, health workers and resort owners. The list of volunteers, health workers and resort owners will be requested from the local government unit. From this list, four ${ }^{4}$ focus groups will be formed consisting of about six to eight participants per group. The information that can be gathered from the focus group participants will enhance the data derived from the key informant interviews.

The sampling design to be used for the community survey is systematic random sampling which relies on arranging the target population according to a predefined ordering scheme and then selecting respondents at regular intervals through that ordered list. The sample size needed for the study is 357 ( $\mathrm{z}=1.96$ based on CI of $95 \%$, absolute precision $=5 \%$ and design effect $=1.0$ ). Target respondents to be recruited will be interviewed from each sampled household. An eligible respondent is any parent or caregiver aged 21 years or older and must be a resident of the site for at least a year.

\section{Data collection instruments}

A data abstraction form will be employed when conducting the records review. For each barangay in Los Baňos, the form will be used to gather data on drowning mortality between 2015 and 2019 ( 5 years) by variables such as age and sex, aquatic location and activity prior to drowning.

Interview guides (interview questions with probing questions) will be utilised for the key informant interviews (online supplemental file 1) and focus group discussions (online supplemental file 2). The interview guide will be used to collect demographic information of the key informants as well as information regarding the key informants' perceptions of severity, causes, prevention and control measures for drowning in the area. The guide will also collect the key informants' knowledge of risk factors, health system capability to address the drowning problem and treatment of those who drown; existing initiatives related to drowning prevention; availability of resources for drowning prevention initiatives and policies to prevent drowning in the area.

Audit tools will be used to collect data from household and community observations. These audit tools will gather data focusing on the presence of water hazards (such as wells, manholes, swimming pools, natural water bodies such as rivers or lakes among others), infrastructure to restrict access to water hazards (such as well covers or barriers) and warning signs around water hazards, the presence of equipment such as flotation devices, presence of lifeguards and adult supervision of children around water hazards. Separate forms are used for households (online supplemental file 3 ) and locations in the broader community (online supplemental file 4).

A community survey tool will also be used to gather quantitative data (table 2). The survey will be utilised to gather information on household drowning experiences. Questions on knowledge and awareness will revolve around prevention of drowning, places where children can drown, treatment facility for those who drown and programmes, activities and policies on drowning prevention. The section on practices will focus on child supervision while at home, while swimming, and while near bodies of water, use of flotation devices especially while on water transport, performing cardiopulmonary resuscitation and swimming skills. The tool will also assess the attitude of the respondents toward adult supervision of children, wearing of flotation devices, drowning prevention, education and information dissemination activities (online supplemental file 5). The survey questionnaire to be utilised in this study is an expanded version of the tool validated and utilised by the lead investigator (JPG) in a pilot project on drowning in the Northern Philippines. ${ }^{36}$

\section{Data gathering procedure}

A letter informing the local health officials in the selected site about the study will be submitted. Once local health officials have given their permission to implement the study, data collection will commence. If clarification is requested by the officials, a discussion or presentation will be scheduled so that the contents and merits of the study can be presented.

Informed consent will be obtained from respondents (key informants, focus groups participants and community survey respondents). Target respondents will be required to sign the consent form prior to data collection. Those who opt not to participate are free to return the consent form unsigned and will not be questioned further. 
Table 2 Sections and specific contents of the community survey tool

\begin{tabular}{|c|c|}
\hline Section of the community survey tool & Items in the community survey tool \\
\hline \multirow[t]{4}{*}{ Personal and household information } & Age \\
\hline & Sex \\
\hline & Occupation \\
\hline & Home ownership \\
\hline \multirow[t]{3}{*}{ Household drowning experience } & Fatal/non-fatal drowning incidents \\
\hline & Activities during the incident \\
\hline & Rescue efforts done \\
\hline \multirow[t]{4}{*}{ Practices } & Child supervision (near bodies of water, while swimming, near wells, etc.) \\
\hline & Use of water transport vehicle \\
\hline & Cardiopulmonary resuscitation \\
\hline & Swimming \\
\hline \multirow[t]{6}{*}{ Attitudes } & Child supervision \\
\hline & Use of water transport vehicle \\
\hline & Use of flotation devices \\
\hline & Participation in drowning prevention activities \\
\hline & Cardiopulmonary resuscitation \\
\hline & Swimming \\
\hline Knowledge and awareness & Drowning prevention \\
\hline
\end{tabular}

For the community survey, the procedures outlined when the systematic random sampling technique is used (ie, the starting point will be chosen randomly and then a table of random numbers will be used to select the next household) will be followed. Target respondents to be recruited will be interviewed from each sampled household. An eligible respondent is any parent or caregiver aged 21 years of age or older and should have been a resident of the site for at least a year.

For the key informant interviews, the date, time and venue to be suggested by the informants for the conduct of the interviews will be followed. Permission to audio record the interviews will be sought from the informants. For the focus group discussion, participants will be asked about their preferred date, time and venue for the activity. Permission to audio record the discussion will also be sought from the focus group participants (figure 3).

For the household and community observations, the lead author, together with a research assistant who are both Filipino, will perform the observation simultaneously. The household to be assessed will be the household of the respondent who participated in the community survey. Community observers will take note all water bodies and potential water bodies (eg, rivers, wells, drains). They will also be asked to identify other hazards that may increase the risk of drowning (such as roads which cross waterways, lack of barriers around wells). Observations will be conducted for $15 \mathrm{~min}$ and the remarks section of the household observation audit tool will be completed by each observer. For community observations, both the lead author and the research assistant will conduct the observations, each community setting will be observed for at least $30 \mathrm{~min}$ and descriptive notes recorded in the remarks section of the community observation audit tool. These notes will be entered independently by each observer.

\section{Plan of analysis}

\section{Epidemiologic data}

Drowning mortality data obtained from the mortality records at the municipal health office will be used to calculate the drowning mortality rate for the site. A range of analytical methods will be used to explore this data 


\section{Technical and Ethical Approval of Study protocol}

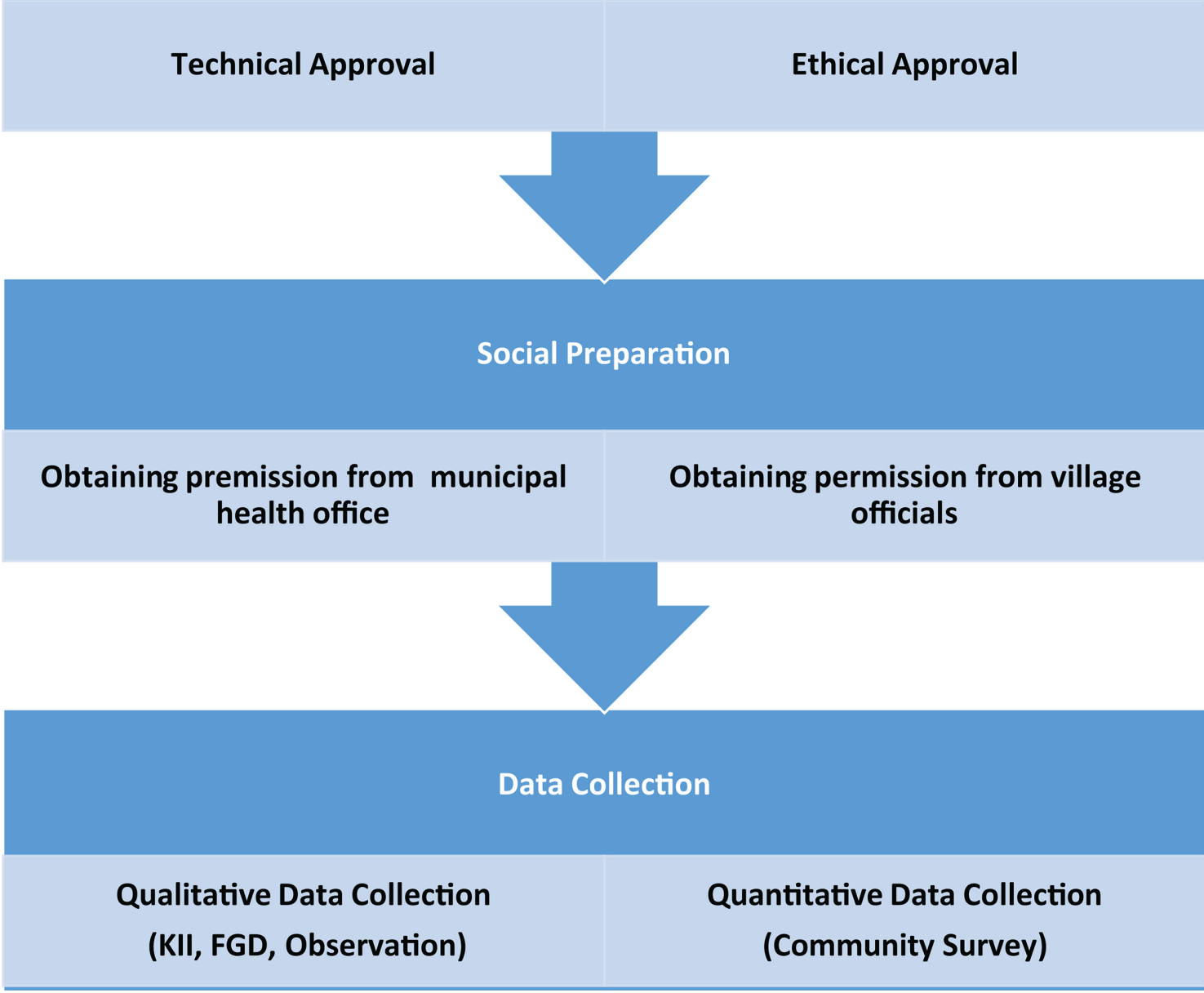

Figure 3 Data gathering procedures.

including analysis of variance, $\chi^{2}$, univariate analysis, relative risk, population rate by age and sex.

Focus groups and key informant interviews

For the qualitative data analysis, key informant interviews and focus group discussions will be transcribed and, where required, translated into English. Thematic analysis will be conducted for the qualitative data using an inductive approach as outlined by Braun and Clarke. ${ }^{37}$ This fivephase methodology will be undertaken as follows: authors will familiarise themselves with the data by rereading the data and noting down initial themes (phase 1). Authors then will individually generate initial themes by systematically coding interesting features of the entire data set (phase 2). The authors will then review the themes generated, solidify and develop a thematic map for coding. All authors will cross-check and confirm individual thematic coding of themes (phase 3). Separately, authors will analyse the qualitative data into the themes (phase 4). All authors will meet to compare the contents of each theme and refine any outstanding issues (phase 5).
Household and community observations

Results coming from the household and community observation will provide the much-needed data on environmental factors contributing to drowning injury. Environmental data will be presented using frequency and percentage distribution tables. The description written by the two independent observers under the remarks section will be discussed and a more detailed descriptive picture of the result of the observation will be presented. Descriptive field notes will also be taken. To perform the observation the Observer will stop and scan the environment taking their time in a $360^{\circ}$ arc. They will also be able to add to these observations as they move through the community to build a full profile.

\section{Community survey}

Descriptive statistics will be used in the analysis of survey data, including exploration of data by age and sex. Using Epi Info V. $6^{38}$, tables and figures will be generated for the different variables of concern. Quantitative data, 
specifically frequency, proportion and measures of central tendency (mean, median, mode) will be presented.

Triangulation of both quantitative and qualitative data will be performed to get a complete picture of the risk factors to be addressed by the drowning prevention programme. The quantitative data from the community survey and themes drawn from qualitative data will be placed into the first four phases of the PPM (figure 1).

\section{Informed consent process}

The informed consent form (ICF) to be used in obtaining informed consent from the key informant, focus group participants and community survey participants contains details about the investigator, purpose of the research, type of intervention, participant selection, voluntary participation, procedures, duration, risks and benefits, incentives/reimbursement, confidentiality, sharing of study results, right to refuse or withdraw, compliance with Philippines Data Privacy Act of 2012, validity of the ICF, expected number of participants and contact details of the investigator and University of the Philippines Manila Research Ethics Board (UPM REB) panel 2.

\section{Public and patient involvement}

There are no patients involved in this study. The public are not involved in the choice of the study location nor the decision to apply the PPM model. The public, in the form of the local community, will be involved in the data collection process as well as being informed of the findings and involved in the design of the chosen intervention. As has been seen in other countries, the involvement of the community is vital to enhancing success of drowning prevention interventions. ${ }^{21} 22$

\section{Ethics approval}

The study protocol has obtained ethical clearance (UPMREB 2017-425-01) from the University of the Philippines Manila Research Ethics Board (UPMREB).

\section{Study team}

The study team is led by a Filipino researcher who has conducted studies on drowning prevention in the past 10 years. $^{12} 1316193639$ He will also lead data collection since the interviews and focus group discussions will be done in the vernacular (Filipino) to ensure study participants understand the main issues being asked in the study. The co-authors have published extensively on drowning and drowning prevention in Australia ${ }^{40} 41$ and globally ${ }^{8} 42$ and have collaborated with the lead author on drowning research. ${ }^{12} 1316$ Co-authors are experienced in conducting mixed-methods studies ${ }^{43}$ and will also be able to contribute skills in data analysis and development of intervention and strategies. Furthermore, the third author is a visiting professor in the institution of the lead author.

\section{DISCUSSION}

Drowning is a threat to public health globally ${ }^{11}$ and a significant problem in the Philippines, ${ }^{12}{ }^{16}$ as it is in other LMICs. $^{811}$ There is a dearth of quality data on the scale and nature of the global drowning burden to inform prevention efforts. ${ }^{1}$ Guided by the PPM, risk factors for drowning will be identified, objectives will be set and strategies will be described and implemented for the identified target audience that will contribute to the reduction of the burden of drowning.

This study will represent one of the first times the PPM, a well-published and widely used model for planning interventions, ${ }^{2324}$ has been applied to the issue of drowning and its prevention. ${ }^{30}$ This study will provide direction in the use of mixed methods of data collection, as well as guidance on the application of the PPM to drowning prevention more broadly across both high income and LMIC contexts. Therefore, this study, and the protocol, represents a useful addition to the literature.

The application of the PPM to the village with the highest drowning mortality rate will assist in the identification of risk factors for drowning including exploring non-fatal drowning (data on which is currently unavailable in the Philippines).$^{16}$ Localised differences mean that local data and insights from key stakeholders must inform local solutions to drowning problems in the community. A community-driven approach to drowning prevention has proven to be successful in Bangladesh and Thailand ${ }^{2122}$ and it is hoped it will also be successful in the Philippines.

The PPM is not just a planning model but an evaluation model as well. ${ }^{23}$ It is to be noted that evaluation is included in the model and different levels of evaluation are covered (process, impact and outcome). If all the steps in the model are utilised, those implementing drowning prevention intervention(s) will be able to assess effectiveness of the intervention(s) based on the evaluation indicators and measures included in the model. Using the PPM will also help to build local capacity, understanding and ownership in the development of interventions to prevent drowning.

A study of this kind is very important for the Philippines for a number of reasons: (i) there is a dearth of research in the field of drowning prevention and this is an important addition to the body of knowledge in this field, (ii) this study is in support of the Multisector Action Plan on Drowning Prevention (Philippines): $2016-2026^{16}$ and (iii) this study follows the recommendations of the WHO specifically strategy 10: research: advance drowning prevention through data collection and well-designed studies. ${ }^{1}$

Drowning is a significant issue in the Philippines and other LMICs. There is a need to gather data on drowning burden including both risk and protective factors to inform prevention efforts. The PPM provides a health promotion framework for this study. In applying the PPM to drowning, it is hoped that this study will 
provide guidance for other nations to apply the PPM to the issue of drowning in their countries.

\section{Author affiliations}

${ }^{1}$ Department of Health Promotion and Education, University of the Philippines Manila, Manila, Philippines

${ }^{2}$ School of Population Health, UNSW Sydney, Kensington, New South Wales, Australia

${ }^{3}$ College of Public Health, Medical and Veterinary Sciences, James Cook University, Townsville, Queensland, Australia

${ }^{4}$ Royal Life Saving Society - Australia, Broadway, New South Wales, Australia

\section{Twitter Amy E Peden @amyepeden and Richard Charles Franklin @Franklin_R_C}

Acknowledgements We acknowledge the lives lost due to drowning and the need to prevent future incidents. We also acknowledge the University of the Philippines for their support.

Contributors JPG conceptualised the study. JPG, AEP and RCF designed the study. JPG will conduct data collection and analyses and RCF and AEP will interpret the findings. JPG, AEP and RCF drafted and critically revised the manuscript. All authors reviewed and approved the final manuscript as submitted.

Funding This work was funded by the UP System Enhanced Creative Work and Research Grant (ECWRG 2016-1-002).

Map disclaimer The depiction of boundaries on this map does not imply the expression of any opinion whatsoever on the part of BMJ (or any member of its group) concerning the legal status of any country, territory, jurisdiction or area or of its authorities. This map is provided without any warranty of any kind, either express or implied.

Competing interests None declared.

Patient consent for publication Not required.

Provenance and peer review Not commissioned; externally peer reviewed.

Supplemental material This content has been supplied by the author(s). It has not been vetted by BMJ Publishing Group Limited (BMJ) and may not have been peer-reviewed. Any opinions or recommendations discussed are solely those of the author(s) and are not endorsed by BMJ. BMJ disclaims all liability and responsibility arising from any reliance placed on the content. Where the content includes any translated material, BMJ does not warrant the accuracy and reliability of the translations (including but not limited to local regulations, clinical guidelines, terminology, drug names and drug dosages), and is not responsible for any error and/or omissions arising from translation and adaptation or otherwise.

Open access This is an open access article distributed in accordance with the Creative Commons Attribution Non Commercial (CC BY-NC 4.0) license, which permits others to distribute, remix, adapt, build upon this work non-commercially, and license their derivative works on different terms, provided the original work is properly cited, appropriate credit is given, any changes made indicated, and the use is non-commercial. See: http://creativecommons.org/licenses/by-nc/4.0/.

\section{ORCID iDs}

Jonathan P Guevarra http://orcid.org/0000-0002-3561-5131

Amy E Peden http://orcid.org/0000-0002-6424-1511

Richard Charles Franklin http://orcid.org/0000-0003-1864-4552

\section{REFERENCES}

1 World Health Organization. Preventing drowning: an implementation guide. Geneva: World Health Organization, 2017.

2 Peden AE, Franklin RC, Leggat PA. Fatal river drowning: the identification of research gaps through a systematic literature review. Inj Prev 2016;22:202-9.

3 Wallis BA, Watt K, Franklin RC, et al. Interventions associated with drowning prevention in children and adolescents: systematic literature review. Inj Prev 2015;21:195-204.

4 Taylor DH, Peden AE, Franklin RC. Next steps for drowning prevention in rural and remote Australia: a systematic review of the literature. Aust J Rural Health 2020;28:530-42.

5 Cenderadewi M, Franklin RC, Peden AE, Devine S, et al. Fatal intentional drowning in Australia: a systematic literature review of rates and risk factors. PLoS One 2017;15:e0231861.
6 van Beeck EF, Branche CM, Szpilman D, et al. A new definition of drowning: towards documentation and prevention of a global public health problem. Bull World Health Organ 2005;83:853-6.

7 Peden AE, Mahony AJ, Barnsley PD, et al. Understanding the full burden of drowning: a retrospective, cross-sectional analysis of fatal and non-fatal drowning in Australia. BMJ Open 2018;8:e024868.

8 Franklin RC, Peden AE, Hamilton EB, et al. The burden of unintentional drowning: global, regional and national estimates of mortality from the global burden of disease 2017 study. Inj Prev 2020;26:i83-95.

9 Peden AE, Franklin RC, Mahony AJ, et al. Using a retrospective cross-sectional study to analyse unintentional fatal drowning in Australia: ICD-10 coding-based methodologies verses actual deaths. BMJ Open 2017;7:e019407.

10 Beerman SB, Bierens J, Clemens T. Clarification and categorization of non-fatal drowning: a draft position statement for review and input by the global drowning community. Geneva: World Health Organization, 2018. https://www.who.int/docs/default-source/ documents/drowning/non-fatal-drowning-categorization.pdf?sfvrsn= 44d18cc1_2

11 World Health Organization. Global report on drowning: preventing a leading killer. Geneva: World Health Organization, 2014.

12 Guevarra JP, Franklin RC, Peden AE. "I want to see a drowningfree philippines": a qualitative study of the current situation, key challenges and future recommendations for drowning prevention in the philippines. Int J Environ Res Public Health 2021;18:381.

13 Guevarra JP, Franklin RC, Basilio JA, et al. Child drowning prevention in the Philippines: the beginning of a conversation. Int $J$ Inj Contr Saf Promot 2015;22:243-53.

14 Department of Health. Administrative order no. 2014 - 0002: revised policy on policy on violence and injury prevention, 2014. Available: https://www.doh.gov.ph/violence-and-injury-prevention-program

15 Lim-Quizon MC, Linnan M. Philippine national injury survey (PNIS) 2004. Manila, Philippines Philippine National Injury Survey; 2008.

16 Guevarra JP, Peden AE, Orbillo LL, et al. Preventing child drowning in the Philippines: the need to address the determinants of health. Children 2021;8:29.

17 Boquet Y, ed. Moving around the Philippines : challenges and dynamics of mobility in a developing country. 17th international conference of the Hong Kong Society for Transportation Studies (HKSTS). Hong-Kong, China: Hong Kong Society for Transportation Studies, 2012.

18 Acosta LA, Eugenio EA, Macandog PBM, et al. Loss and damage from typhoon-induced floods and landslides in the Philippines: community perceptions on climate impacts and adaptation options. International Journal of Global Warming 2016;9:33-65.

19 Martinez RE, Go JJ, Guevarra J. Epidemiology of drowning deaths in the Philippines, 1980 to 2011. Western Pac Surveill Response J 2016;7:1-5.

20 Philippines Department of Health. Violence and injury prevention program: department of health, Philippines, 2020. Available: https:// www.doh.gov.ph/violence-and-injury-prevention-program

21 Rahman F, Bose S, Linnan M, et al. Cost-Effectiveness of an injury and drowning prevention program in Bangladesh. Pediatrics 2012;130:e1621-8.

22 Sansiritaweesook G, Muangsom N, Kanato M, et al. Effectiveness of community participation in a surveillance system initiative to prevent drowning in Thailand. Asia Pac J Public Health 2015;27:NP2677-89.

23 Green L, Kreuter MK. Health program planning: an education and ecological approach. 4th edition. New York: McGraw Hill, 2005.

24 Crosby R, Noar SM. What is a planning model? an introduction to PRECEDE-PROCEED. J Public Health Dent 2011;71 Suppl 1:S7-15.

25 Porter CM. Revisiting Precede-Proceed: a leading model for ecological and ethical health promotion. Health Education Journal 2016:75:753-64.

26 Howat P, Jones S, Hall M, et al. The PRECEDE-PROCEED model: application to planning a child pedestrian injury prevention program. Inj Prev 1997;3:282-7.

27 Hunter K, Keay L, Clapham K, et al. "He's the number one thing in my world": application of the PRECEDE-PROCEED model to explore child car seat use in a regional community in New South Wales. Int $J$ Environ Res Public Health 2017;14:1206.

28 Omidi S, Farmanbar R, Mokhtarpour S. The effect of educational intervention based on PRECEDE-PROCEED model on promoting traffic safety behaviors in primary schools students of Tabriz in 2014. Journal of Education and Community Health 2016;2:48-56.

29 Sezgin D, Esin MN. Effects of a PRECEDE-PROCEED model based ergonomic risk management programme to reduce musculoskeletal symptoms of ICU nurses. Intensive Crit Care Nurs 2018;47:89-97. 
30 Quan L, Shephard E, Bennett E. Evaluation of a drowning prevention campaign in a Vietnamese American community. International Journal of Aquatic Research and Education 2020;12:4.

31 Cinco M. 2 UPLB students drown in Makiling stream: Inquirer. net, 2012. Available: https://newsinfo.inquirer.net/320541/2-uplbstudents-drown-in-makiling-stream

32 Cinco M. Cop dies as zipline SNAPs in Laguna resort: Inquirer.net, 2017. Available: https://newsinfo.inquirer.net/933438/police-ziplineaccident-resort-los-banos-laguna-dead

33 Valle Rey M. Two girls in Laguna drowned in lake after Chased by dog: Philippines news, 2019. Available: https://philnews.ph/2019/12/ 31/two-girls-in-laguna-drowned-in-lake-after-chased-by-dog/

34 O'Mara-Eves A, Brunton G, McDaid D, et al. Community engagement to reduce inequalities in health: a systematic review, meta-analysis and economicanalysis. Public Health Research 2013;1.

35 Etikan I, Bala K. Sampling and sampling methods. Biometrics and Biostatistics International Journal 2017;5:00149.

36 Guevarra J, Albornoz RM, Go JJL. Knowledge, attitudes and practices of parents in an urban coastal community on preventing childhood drowning. Acta Medica Philippina 2010;44:32-9.

37 Braun V, Clarke V. Using thematic analysis in psychology. Qual Res Psychol 2006;3:77-101.
38 Centers for Disease Control and Prevention (CDC). Epi Info 6: centers for disease control and prevention (CDC), 2017. Available: https:// www.cdc.gov/epiinfo/support/downloads/previous/ei6.html

39 Jonathan PG, BASILIO JA, Lita LO, et al. The utilization of the safe community assessment tool and development of community action plan for injury prevention. J Natl Inst Public Health 2013;62:526.

40 Peden A, Franklin RC, Scarr J. Measuring Australian Children's Water Safety Knowledge: The National Water Safety Quiz. International Journal of Aquatic Research and Education 2017;10.

41 Peden AE, Franklin RC, Leggat PA. Developing drowning prevention strategies for rivers through the use of a modified Delphi process. Inj Prev 2020;26:240-7.

42 Peden AE, Franklin RC, Clemens T. Can child drowning be eradicated? A compelling case for continued investment in prevention. Acta Paediatr 2021;110:1-8.

43 Harrison H, Birks M, Franklin R, et al. Case study research: Foundations and methodological orientations. In: Forum qualitative Sozialforschung/Forum: qualitative social research. 18, 2017.

44 Philippines News Agency. Map of Los Baňos, Laguna: Philippines news agency, 2020. Available: https://files.pna.gov.ph/category-list/ 2020/06/28/los-banos-laguna-map.png 